\title{
Enhanced Absorption and $<1 \%$ Spectrum-and-Angle-Averaged Reflection in Tapered Microwire Arrays
}

Sisir Yalamanchili ${ }^{1,3 \dagger}$, Hal S. Emmer ${ }^{1, \dagger}$, Katherine T. Fountaine ${ }^{2,6}$, Christopher T. Chen ${ }^{1}$, Nathan S. Lewis ${ }^{2-5^{*}}$, and Harry A. Atwater ${ }^{1,3,4}$

${ }^{1}$ Division of Engineering and Applied Sciences, California Institute of Technology, Pasadena, CA 91125

${ }^{2}$ Division of Chemistry and Chemical Engineering, California Institute of Technology, Pasadena, CA 91125

${ }^{3}$ The Joint Center for Artificial Photosynthesis, California Institute of Technology, Pasadena, CA 91125

${ }^{4}$ Kavli Nanoscience Institute, California Institute of Technology, Pasadena, CA 91125

${ }^{5}$ Beckman Institute, California Institute of Technology, Pasadena, CA 91125

${ }^{6}$ NGNext, Northrop Grumman Aerospace Systems, 1 Space Park Dr., Redondo Beach, CA 90278

${ }^{\dagger}$ These authors contributed equally

*Corresponding Author: haa@ caltech.edu 
This is a 7 page document with 3 sections ( S1, S2, and S3), and 5 figures (S1-S5)

\section{S1. Transmission Electron Microscopy (TEM)}
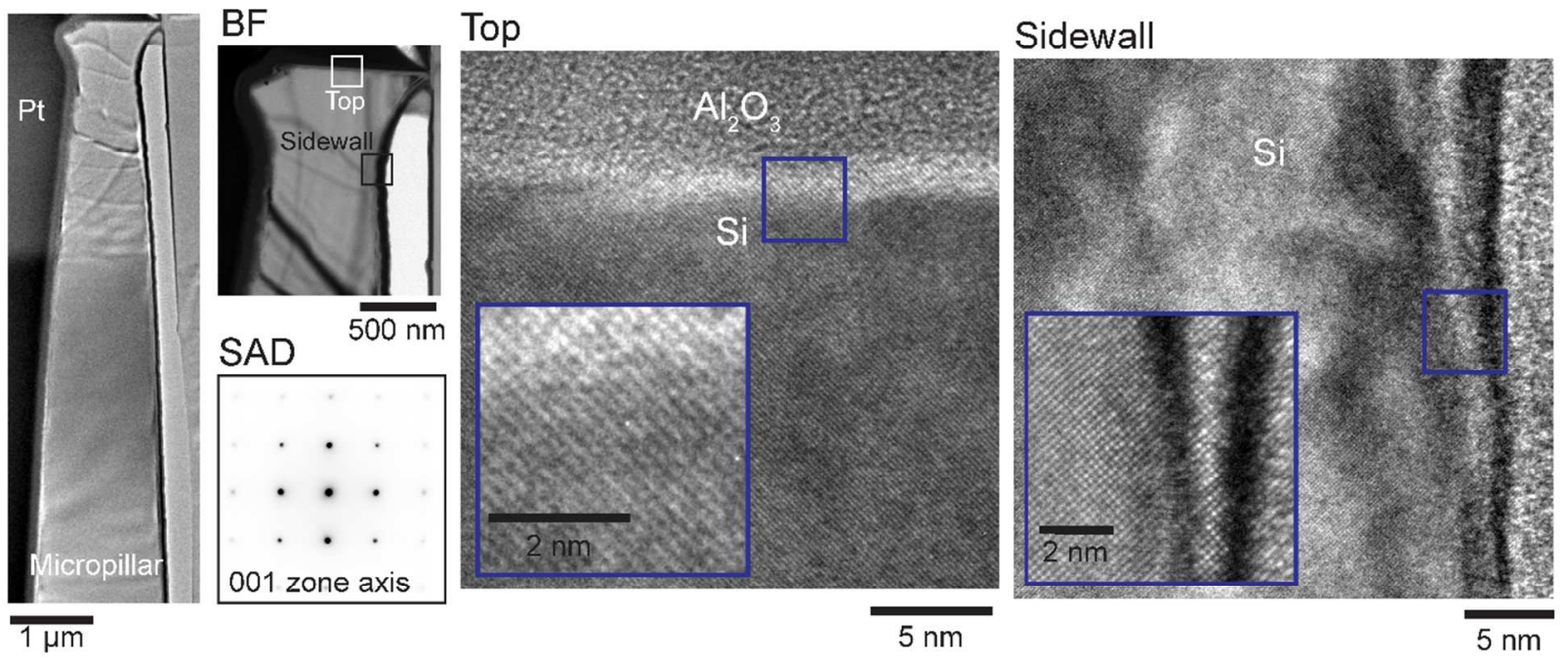

Figure S1: Transmission electron microscopy (TEM) study of a single etched microwire immediately after ICPRIE with the Al2O3 mask intact on top and with no cleaning except a solvent rinse is aligned to the 001 zone axis. A large area corresponding to the tip of a wire was studied (far left). Bright field images (BF) are devoid of contrast within the etched wire other than bend contours introduced during sample preparation, and selected area diffraction (SAD) shows a clean pattern. Higher resolution images at the top of the wire (center) shows no evidence of damage to the wire tip which was protected by the hard mask during growth. However, a high resolution imaging reveals a thin $(\sim 2-3 \mathrm{~nm})$ region on the sidewalls (far right) which could correspond to damage introduced during the etching process. These data were collected at 300 $\mathrm{kV}$ in an FEI Technai TF30 TEM.

Sample preparation for transmission electron microscopy: Etched microwires were removed from the host $\mathrm{Si}$ wafer by mechanical cleaving with a razor blade before being dispersed in 
isopropyl alcohol. The resulting solution was spun onto a Si wafer for $30 \mathrm{~s}$ at $2000 \mathrm{rpm}$. The wire-coated wafer was then coated with $\sim 20 \mathrm{~nm}$ of $\mathrm{Al}_{2} \mathrm{O}_{3}$ with atomic layer deposition. Axial cross sections were made in a FEI Versa dual-beam focused ion beam instrument, extracted with an FEI EasyLift NanoManipulator and welded to an Omniprobe $\mathrm{Cu}$ liftout TEM grid before thinning to electron transparency. 


\section{$\underline{\text { S2. Microwave photoconductivity decay lifetime measurements }}$}

Schematic of the custom built microwave photoconductivity decay (MW-PCD) lifetime measurement setup is shown in Fig. S2. This technique is similar to the widely used quasisteady-state photoconductance technique (QSSPC), except that the photoconductivity decay is obtained by measuring the reflection of a microwave at the sample following a light pulse (1064 $\mathrm{nm}$ for $5 \mathrm{~ns}$ in our case) instead of the permeability change of the sample measured via coupling the sample by a coil to a radio-frequency bridge ${ }^{1}$.

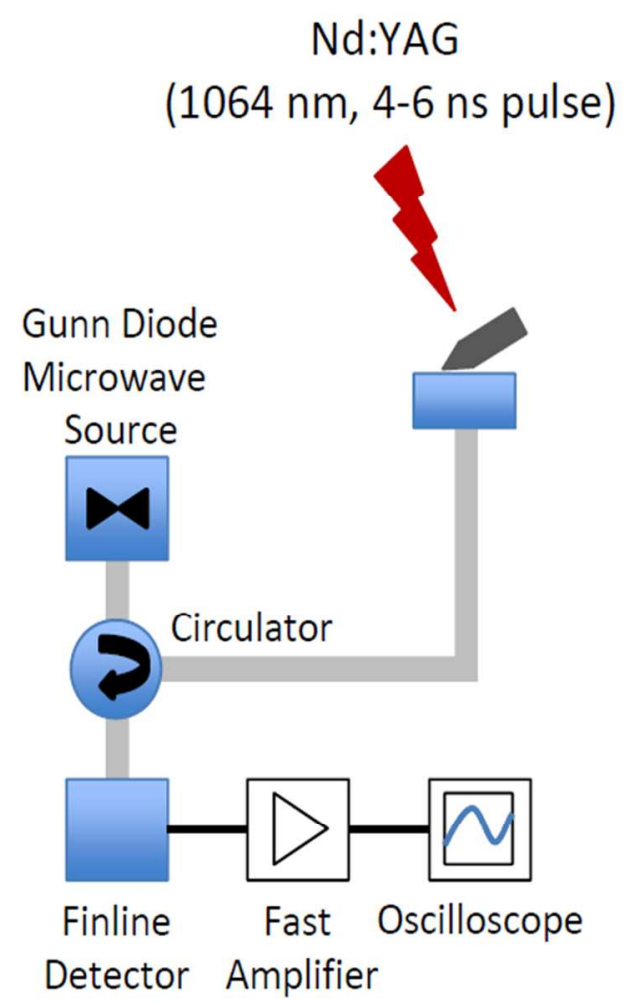

Figure S2: Schematic of home built microwave detected photoconductivity decay setup 


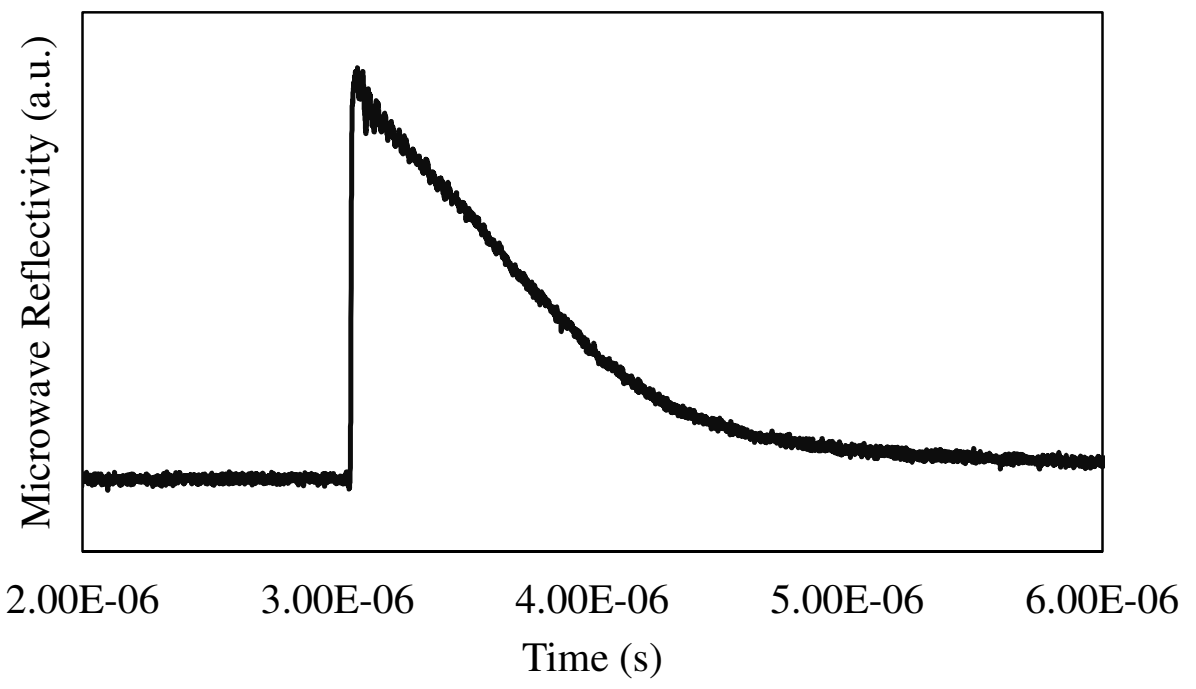

Figure S3: Microwave reflectivity of $\mathrm{ALD} \mathrm{Al}_{2} \mathrm{O}_{3}$ passivated tapered microwire arrays with in situ back surface passivation using $5.8 \mathrm{M} \mathrm{HCl}$ measured using the microwave photoconductivity setup with $0.75 \mu$ s carrier lifetime 


\section{S3. Additional details regarding tapered microwire samples}

Tapered microwires embedded PDMS films: Total thickness of the film is $120 \mu \mathrm{m}$ with $75 \mu \mathrm{m}$ tall tapered microwires embedded in them. The shape of the microwires is very close to a cone $75 \mu \mathrm{m}$ tall, $25 \mathrm{~nm}$ top radius, and $3.5 \mu \mathrm{m}$ bottom radius. The fill fraction of $\mathrm{Si}$ in the film is plotted in Fig. S4 below.

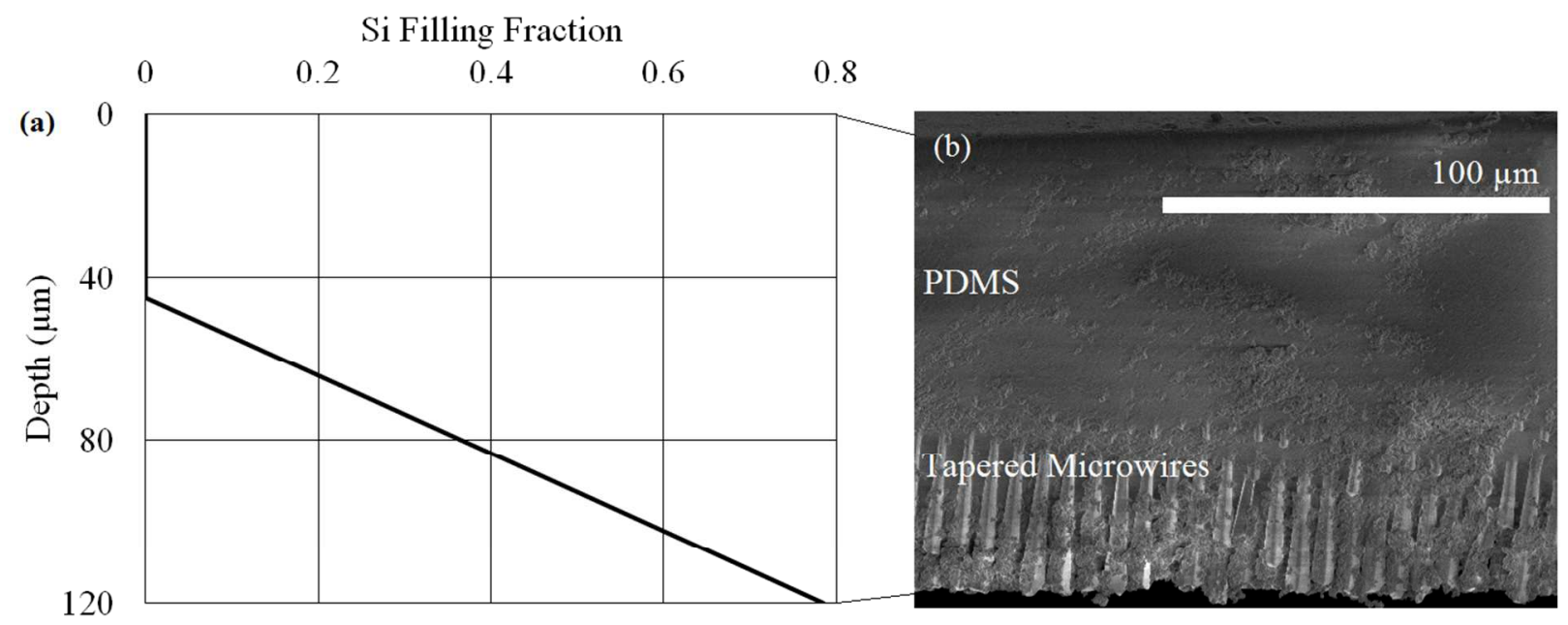

Figure S4: (a) Si fill fraction variation from the top of the peeled off PDMS films with tapered microwires embedded in it. (b) SEM cross section image of the films showing the tapered microwires popping out of PDMS at the bottom of the film. 


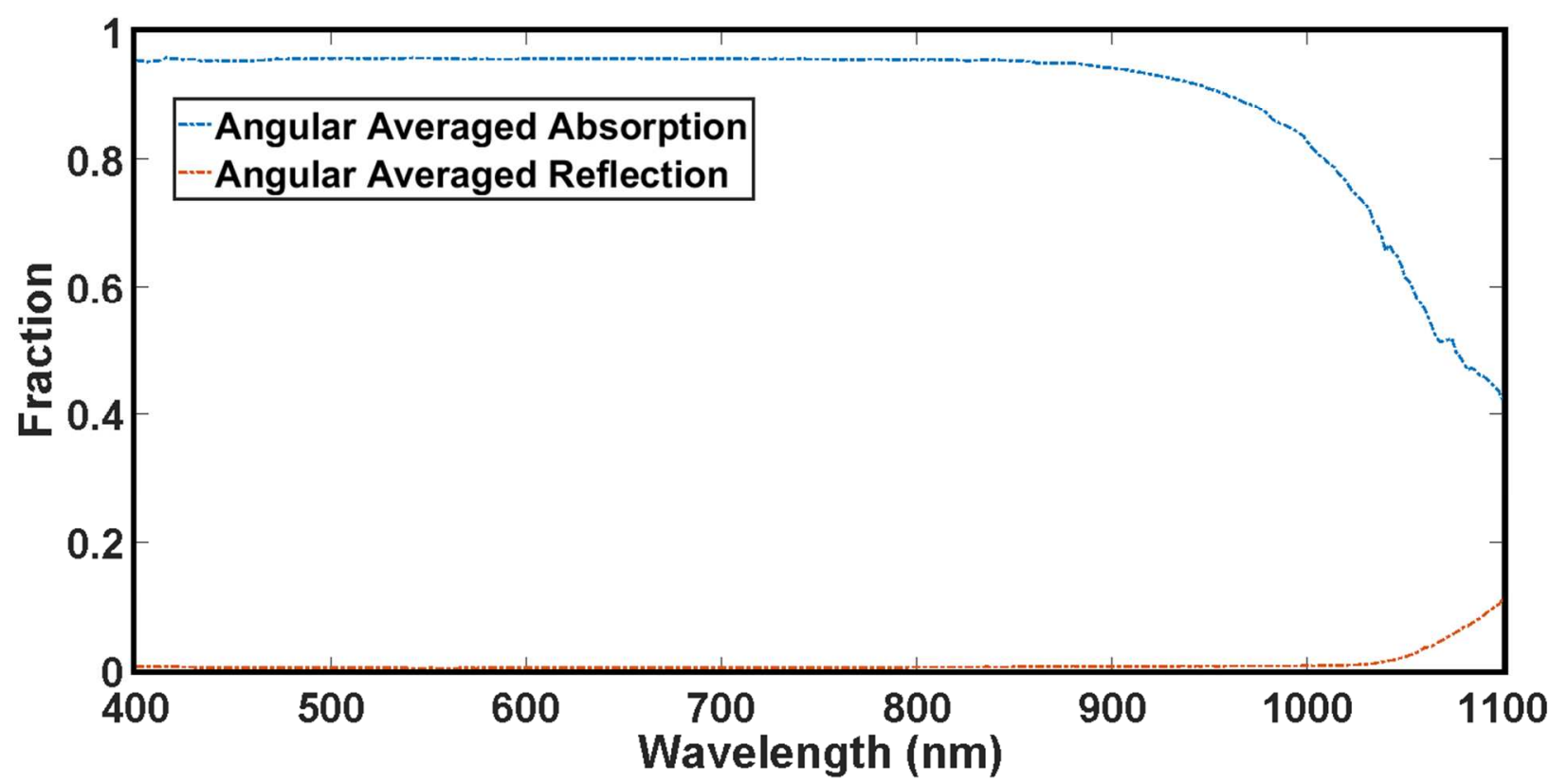

Figure S5: 2D plot of angular averaged reflection from Si substrate with tapered microwires etched into it (in red), and angular averaged absorption in tapered microwire arrays embedded in PDMS (in blue) plotted in $400 \mathrm{~nm}-1100 \mathrm{~nm}$ wavelength range.

(1) Rein, S., Lifetime measurement techniques. In Lifetime Spectroscopy - A method of defect characterization in silicon for photovoltaic applications, Springer: Germany, 2005; pp 59-68. 